\title{
Investigation on the Functionality of Thermo-Responsive Origami Structures
}

\author{
Mehrshad Mehrpouya $^{\mathrm{a}, \mathrm{b}}$ *, Ali Azizi ${ }^{\mathrm{b}}$, Shahram Janbaz $^{\mathrm{c}}$, Annamaria Gisario ${ }^{\mathrm{b}}$ \\ ${ }^{a}$ Department of Industrial Engineering and Management, HZ University of Applied Science, Het Groene Woud 1-3, \\ 4331 NB Middelburg, The Netherlands \\ ${ }^{\mathrm{b}}$ Department of Mechanical and Aerospace Engineering, Sapienza University of Roma, Via Eudossiana 18, 00184 \\ Rome, Italy \\ ${ }^{\mathrm{c}}$ Department of Biomechanical Engineering, Faculty of Mechanical, Maritime, and Materials Engineering, Delft \\ University of Technology, Mekelweg 2, Delft 2628CD, The Netherlands \\ Corresponding e-mail: mehrshad.mehrpouya@hz.nl \\ ORICID: 0000-0001-8939-7937
}

$\square$

\begin{abstract}
Additive manufacturing has recently been introduced as a reliable technique for the fabrication of highly complex geometries that were not possible before. Due to the flexibility in the organization of material properties such as responsive elements in space, additive manufacturing is now a capable technology for the production of smart structures that can transform their geometry, for example, from a compact state to a deployed configuration. Among others, fused deposition modeling (FDM) can reliably be used to manufacture polymeric constructs with high resolution. PLA, the most popular polymer in FDM printing is a shape-memory polymer. Therefore, the manufacturing of shape-transforming constructs can be simplified to the construction of foldable products that can be programmed simply by applying mechanical forces. Origami can then be used as a simple platform in which the shape-transforming of a programmed construct is via the folding of material through the thinner sections (hinges). In our study, we used PLA and FDM additive manufacturing to fabricate foldable structures. We then investigated the effects of different parameters namely total thickness, layer height, nozzle temperature, and activation temperature on the shape recovery of the manually programmed origami structures. Additionally, we have shown that gripping, material-release, and blockage of gaps in engineering problems can be planned based on the unfolding of pyramid-like origami blocks.
\end{abstract}

Keywords: 4D printing, PLA, Shape Memory Polymer, Origami Structures, FDM

This article has been accepted for publication and undergone full peer review but has not been through the copyediting, typesetting, pagination and proofreading process, which may lead to differences between this version and the Version of Record. Please cite this article as doi: $\underline{10.1002 / \text { adem.202000296 }}$ 


\section{WILEY-VCH}

\section{Introduction}

Additive manufacturing ( $\mathrm{AM}$ ), has risen as a flexible innovation platform and has turned into an influential strategy for future modern manufacturing systems [1-3]. Its applications have extended rapidly from visual prototypes in commercial industries (e.g., in aerospace, automotive and biomedical fields) to electronic gadgets, tissue engineering, and high-performance metamaterials [4, 5]. Various methods of additive manufacturing have been developed to meet the demands for the fabrication of complex structures with high resolutions. For example, multi-jet 3D printing can accurately combine digital patterns of soft and hard polymers to fabricate products with a gradient in their mechanical properties [6,7]. Among others, fused deposition modeling (FDM) is the most popular polymer 3D printing method, mainly because of its inexpensiveness and easy handling [8]. This technology is as simple as the extrusion of polymeric filaments through a hot nozzle with a temperature above the melting temperature of the feeding material. The nozzle head movements are according to the planned pattern of printing to fabricate the desired 3D structure in a layer by layer scheme [9].

Although geometry is the key to create unusual functionalities [10] and additive manufacturing seems to be the best solution to fabricate complex shapes but there are still many other interesting functionalities such as shape-shifting of biological systems [11] that are not solely related to the geometry of materials but also based on the development of their constituent materials [12]. In recent years, a new technology called $4 \mathrm{D}$ printing has emerged which seems to be the solution to overcome the limitations of most previous manufacturing techniques. 4D printing refers to the fabrication of smart materials whilst the shape of the 3D printed construct can transform into another predetermined form in response to a stimulus [13]. Nowadays, a popular definition for 4D printing is that the property, functionality, and shape of a 3D printed object could be changed with time when it is triggered by a specified stimulus, such as light [14], water [15], heat [16], $\mathrm{pH}$ [17], etc. 4D printing is the outcome of the fast progression in additive manufacturing of complex constructs and interdisciplinary standpoint towards the design of smart materials [4, 12].

The responsiveness of material to stimuli is the key factor in $4 \mathrm{D}$ printing. Compared to smart metals, smart polymers, normally, exhibit large deformations, therefore, a high level of complexity in shape-shiting is achievable [4]. Furthermore, the versatility in the selection of stimuli-responsive polymers and the simplicity in the manipulation of properties propose them as the most reasonable solution for the manufacturing of smart constructs. Therefore, complex systems with complex patterns of shape-shifting can be produced using additive manufacturing [18] while the stimuliresponsive elements are rationally arranged within their structure. Interestingly, most of the polymers which are used in additive manufacturing show favorable shape memory effect (SME) $[19,20]$. Among other shape-memory polymers, PLA has been proven as the most available and inexpensive polymer that can easily be 3D printed and programmed using FDM [21].

Although the cost of materials used in 4D printing and fabrication is relatively low, the costs related to the design process make it expensive. In this paper, compared to $4 \mathrm{D}$ printing, we propose the manual programming of hinged-based structures as a simple solution for the organization of desired deformations for various applications. As the shape recovery can be the main concern regarding the transformation of the programmed materials, we focus on the time-dependent recovery of the proposed origami-based materials. We, therefore, investigate the effect of four major parameters (i.e., the total thickness of the sample, layer height of the material, printing, and activation temperatures) on the shape-recovery of manually folded/programmed pyramid structure. 


\section{WILEY-VCH}

\section{Materials and method}

An ordinary polylactide (PLA) filament, as a stimuli-responsive polymer, used in this study (diameter $=1.75 \pm 0.05 \mathrm{~mm}$, viscat softening temperature $55-60^{\circ} \mathrm{C}$, manufactured by Filoalfa, Brussels) to produce flat origami structures (see figure 1a) using a hobbyist FDM 3D printer (Meeds-Zaturi equipped with a $0.4 \mathrm{~mm}$ nozzle, Italy). Linear infill pattern with the infill density of 100 percent was chosen. During fabrication, the temperature of the printing bed was set to $40^{\circ} \mathrm{C}$ while the printing speed and the travel speed were $50 \mathrm{~mm} / \mathrm{s}$ and $100 \mathrm{~mm} / \mathrm{s}$ respectively. PLA becomes rubbery and is easy to print while heated above its glass transition temperature $\left(T_{\mathrm{g}}\right)$ [22]. The 3D printed flat origami structures are easy to fold since at high temperatures their hing parts are very soft. The manually folded structures in the form of pyramids can, then, be fixed by cooling down below the $T_{\mathrm{g}}$. Such a PLA structure can recover its original flat shape when heated again above its $T_{\mathrm{g}}[21,23,24]$. It is important to take into account that the stresses remained in the structure of the 3D printed specimens, based on the pattern of printing and possible internal adhesions during the 'manual' programming of flat matter, which will result in some deviation from the as-printed geometry. This paper principally investigates the effects of different factors on the recovery behavior of an origami structure by studying the:

I. Recovery Ratio of the origami structure

II. Unfolding Rate of the origami structure

\subsection{Origami structure}

The initial flat form of the pyramid-shape origami structures was designed with 4 different thicknesses. As it is illustrated in Fig. 1(a), each design involves two main parts namely panel and hinge. The first part is the non-bending part that does not exhibit a major bending or deformation during the programming and shape-recovery procedures. The second part is the bending part that acts as a hinge during programming and shape-recovery of the origami pyramids. The width of the hinge parts in all the four designs is $1 \mathrm{~mm}$. The hinges are used to integrate four identical equilateral triangle panels into a $20 \times 20 \mathrm{~mm}$ square base. The thickness of the hinge and panel parts of the four designs are presented in a table in Fig. 1a as well.

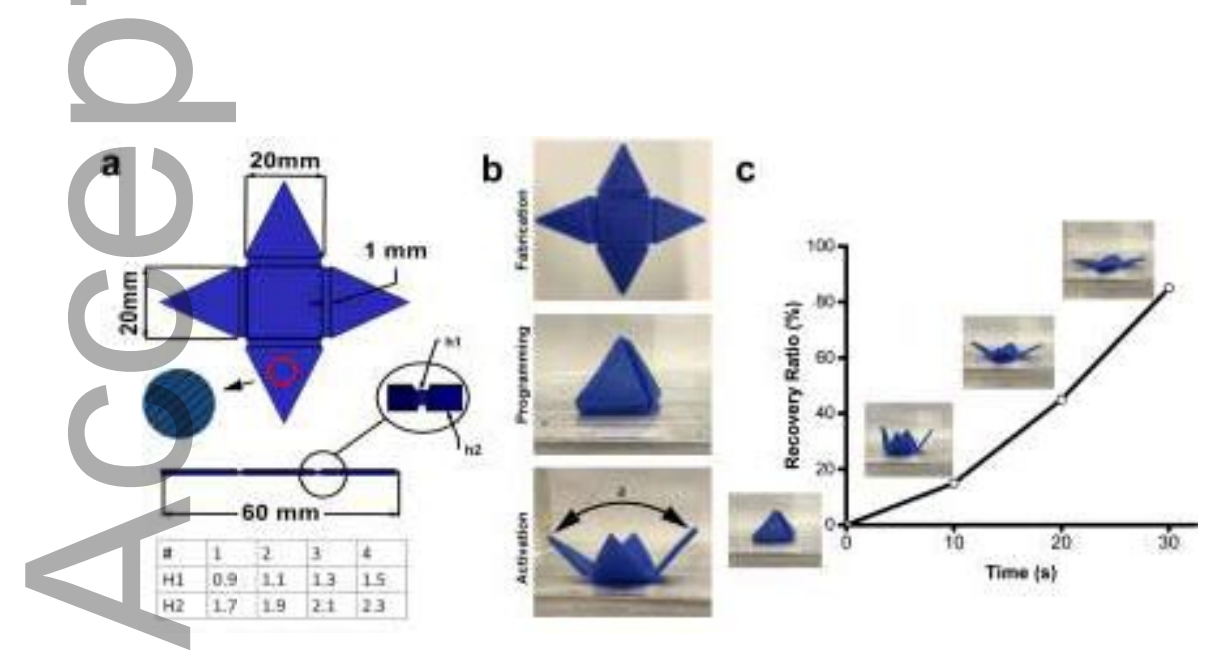

Figure 1. (a) The schematic of the origami design and a table defining the thicknesses for bending and non-bending parts. The inset displays the infill pattern of the printed sample with a raster angle of $45^{\circ}$. (b) The initial flat shape of a $3 \mathrm{D}$ printed sample, programmed pyramid shape, and the recovery at a temperature over $\mathrm{Tg}$. (c) The time-dependent recovery process of a programmed pyramid at $70^{\circ} \mathrm{C}$. 


\section{WILEY-VCH}

Figure $1 \mathrm{~b}$ represents an origami specimen with a permanent flat shape. During the manual programming, the flat structure is folded into a pyramid-like structure. Before folding, the temperature of the hot water bath used in our experiments was set to $65^{\circ} \mathrm{C}$ (n.b., the $T_{\mathrm{g}}$ of PLA ranges from $62-63^{\circ} \mathrm{C}$ ). Then, the samples were put inside the water for 60 seconds [25], and immediately after that, they were transformed into a pyramid form using external forces. Specimens were then taken out of the hot water bath and let to cool down at ambient temperature while the external loads are still maintained. The subsequent removal of the external loads does not affect the configuration of the specimens meaning that the material is programmed (Fig 1b). To recover the flat shape, the shape memory material requires to be heated again above its glass transition temperature (Fig 1b,c). We studied the effect of three temperature values $\left(65^{\circ} \mathrm{C}, 70^{\circ} \mathrm{C}, 75^{\circ} \mathrm{C}\right)$ on the shape recovery of the representative origami structures. Figure 1c illustrates the recovery process of a closed origami sample through the activation.

Furthermore, we studied the recovery ratios and the unfolding rates (degree/s) for the specimens with different parameters. We used a digital camera to track the unfolding process during activation. Moreover, photos were taken from the final configuration of samples in hot water and an image processing tool, ImageJ software, was used to estimate the angle $(\alpha)$ between the panels of the structure. For analyzing the unbending rate of samples, the recorded videos were analyzed using Tracker software.

\subsection{Printing parameters}

The effect of four main parameters (i.e., total thickness: $0.9-1.5 \mathrm{~mm}$, layer height: $0.15-0.30 \mathrm{~mm}$, nozzle temperature: $175-225{ }^{\circ} \mathrm{C}$, and activation temperature: $65-75{ }^{\circ} \mathrm{C}$ ) on the recovery of programmed pyramids was studied separately. Each aspect was evaluated based on three similar experiments to assess the effect of variations during fabrication, programming, and activation of pyramid structures. Figure $2 \mathrm{a}-\mathrm{d}$ show the values used in our experiments.

\section{Results and discussion}

\subsection{Recovery ratio}

The trend of recovery ratio (unfolding-angle/folding-angle) and unfolding angle are presented in Fig. 2e-h based on various experimental parameters. It is observed that slightly above the glass transition temperature of PLA $\left(62-63^{\circ} \mathrm{C}\right)$ an $85 \%$ recovery is achieved (Figure 2e). Increasing the activation temperature up to $75^{\circ} \mathrm{C}$ results in full shape recovery. The minimum activation temperature, $65^{\circ} \mathrm{C}$, restricts the recovery ratio, however, the higher printing layer height maximizes the shape recovery up to $90 \%$ (Figure 2f). As the total thickness of the samples is constant, increment in printing layer thickness results in fewer air gaps in the structure of hinges, therefore, the chance of deviation from $100 \%$ recovery based on the possible imperfections becomes less.

It is important to consider the higher printing layer thickness increases the homogeneity in the structure of the 3D printed flat material, however, it will negatively affect the quality of the surfaces. This is of importance when the geometry of the surface plays an important role in defining physical and biological functionalities. Higher nozzle temperature can similarly increase the homogeneity of the 3D printed constructs since it can strongly reduce the viscosity of the melted PLA and, therefore, allow a better diffusion of successive extrudes PLA in the underlying layer. Within the recommended range of nozzle temperature $\left(175-225^{\circ} \mathrm{C}\right)$, a $10 \%$ increase in the recovery ratio is observed as the temperature increases to $225^{\circ} \mathrm{C}$ (Figure $2 \mathrm{~g}$ ). Opposite to the printing parameters and activation temperature, the increase in the total thickness of the specimens has a negative effect on shape recovery. Increasing the total thickness of specimens up to $1.5 \mathrm{~mm}$ (from 9 $\mathrm{mm}$ ) result in a 5\% less recovery ratio. This can correspond to the higher accumulation of imperfections and permanent deformations during the process of programming (Figure $2 \mathrm{~h}$ ). 


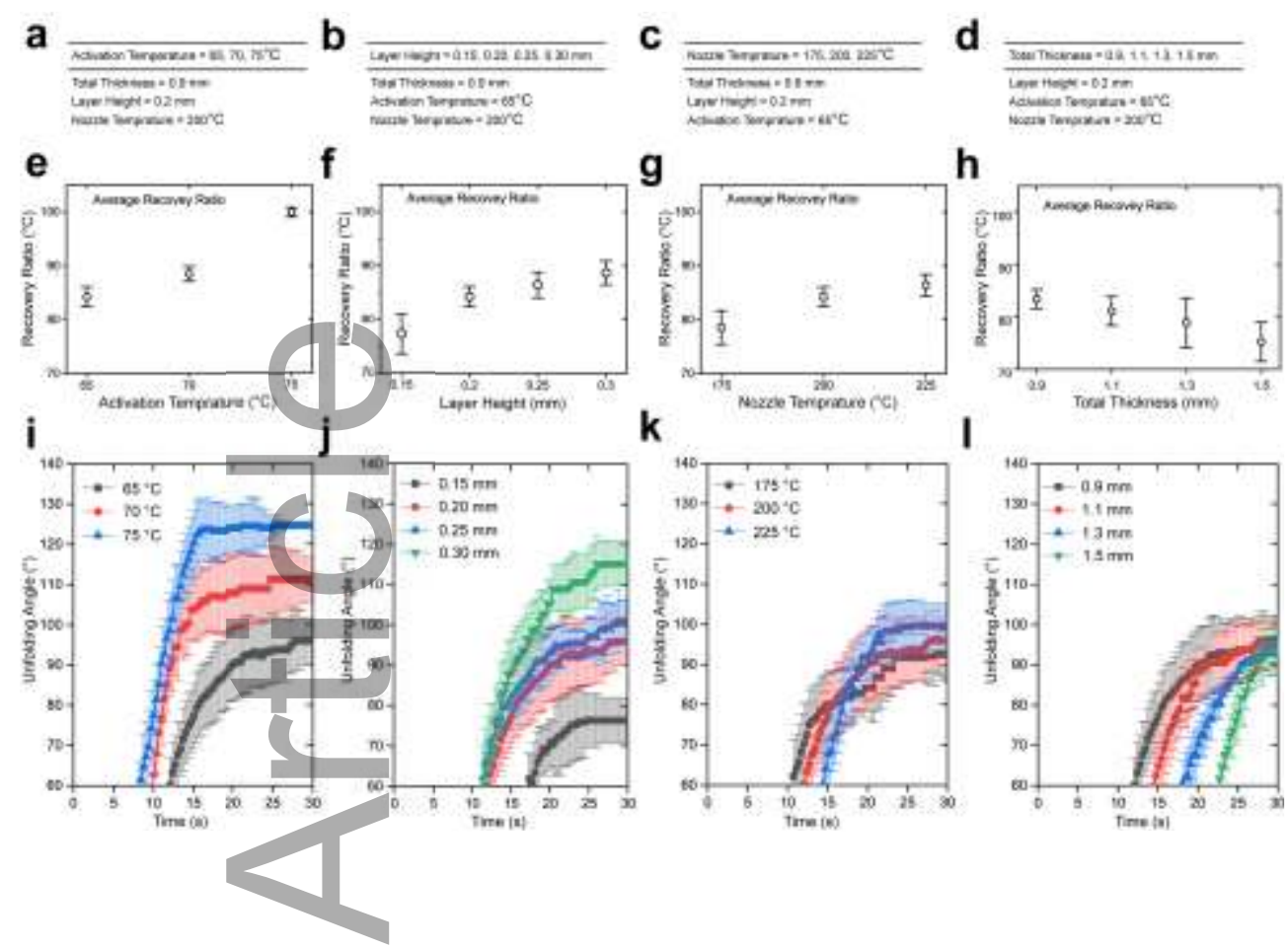

Figure 2. The effect of the experimental parameters on the recovery ratio and unfolding angle of the printed origami samples. Parameters to study the effect of (a) activation temperature, (b) layer heights, (c) nozzle temperatures, and (d) total thickness. (e-h) The recovery ratio of the samples for different activation temperatures, layer heights, nozzle temperatures, and total thickness. (i-l) The unbending angle of the samples for different activation temperatures, layer heights, nozzle temperatures, and total thickness.

\subsection{Unbending angle}

Shape recovery of PLA specimens is a time-dependent process since heat transfer and viscoelastic behavior of PLA (at temperatures above $\mathrm{Tg}$ ) prevent a rapid shape-recovery. Among the other parameters, activation temperature has a significant influence on the shape recovery of origami structures (Figure 2i). This due to the higher rate of heat transfer in the hinges and the lower viscosity of PLA at higher temperatures. Furthermore, the full recovery only observed for the activation temperature $75^{\circ} \mathrm{C}$. It is important to state that the nominal glass transition temperature represents the average temperatures that allow the transition from glassy to a rubbery behavior regarding different phases of PLA. Close to the glass transition temperature of PLA a maximum of $75 \%$ recovery absorbed. The other interesting point to be mentioned is that the response time to the stimulus does not vary so much in diverse temperatures, thus, it can be concluded that the initial penetration of the heat in the samples mainly depends on the structure of the model rather than the amount of the heat; while the final effect on the behavior of shape recovery can be influenced by the amount of the heat (Figure 2i).

Figure $2 \mathrm{j}$ illustrates the unbending rate of the samples with similar total thicknesses but different layer heights. As seen in the graph, layer height has more influence on the speed of unbending rather than on the activation time so that the smaller layer height has a slower unfolding procedure according to the inclination of graphs. The response time of samples has an inverse relationship with the layer height; in other words, the sample with the least layer height responds to stimulus later than the sample with thicker layer height. It can be caused by having more layers and more air gaps in the samples with less layer height and heat need more time to penetrate and affect the sample to react. Nozzle temperature makes small differences in the response time of samples to the 


\section{WILEY-VCH}

stimulus in the unfolding stage (Figure $2 \mathrm{k}$ ). It can be seen that the rate of unbending is almost similar in all the specimens initially, nevertheless, this rate decreased earlier in samples printed with lower nozzle temperature which causes less recovery efficiency as discussed before. Samples that were printed with a higher nozzle temperature react to the stimulus a little later than the ones printed with lower nozzle temperature.

Finally, Figure 21 shows that the total thicknesses can effectively influence the activation time, however, the maximum recovery is almost reached at a similar time $(30 \mathrm{sec})$. It is obvious that as samples get thickened their response to stimulus takes a longer time. The thickest hinge in this study responds about 7 seconds later than the thinnest hinge to heat in comparison with the thinnest sample. This is beneficial while a sequential bending/unbending is desired.

The design of hinged-base structures and the manual programming of origami systems can serve as a simple platform for organizing complex but enough accurate functions with various applications such as applications in biomedical and surgical devices [26] and soft robotics [27, 28]. We have demonstrated that the very basic origami structure used in our study can serve different objects such as delivery or transplantation of (bio-) materials, grasping of objects, and plugging the defects. A similar strategy can be used in the design of smart grippers, switches, sensors, or actuators. Figure 3 shows the application of the 3D printing process for fabricating an origami structure which can be used for material delivery, serve as a smart gripper and stimuli-responsive blocking object. It is important to mention that the very complex actions can be organized based on the geometrical design of basic elements and the relevant activation rates and delays. Moreover, the activation temperature might be a concern while the origami constructs are aimed to be used inside the human body, therefore, the parameters regarding the manufacturing process can optimize the performance of the aimed actions.

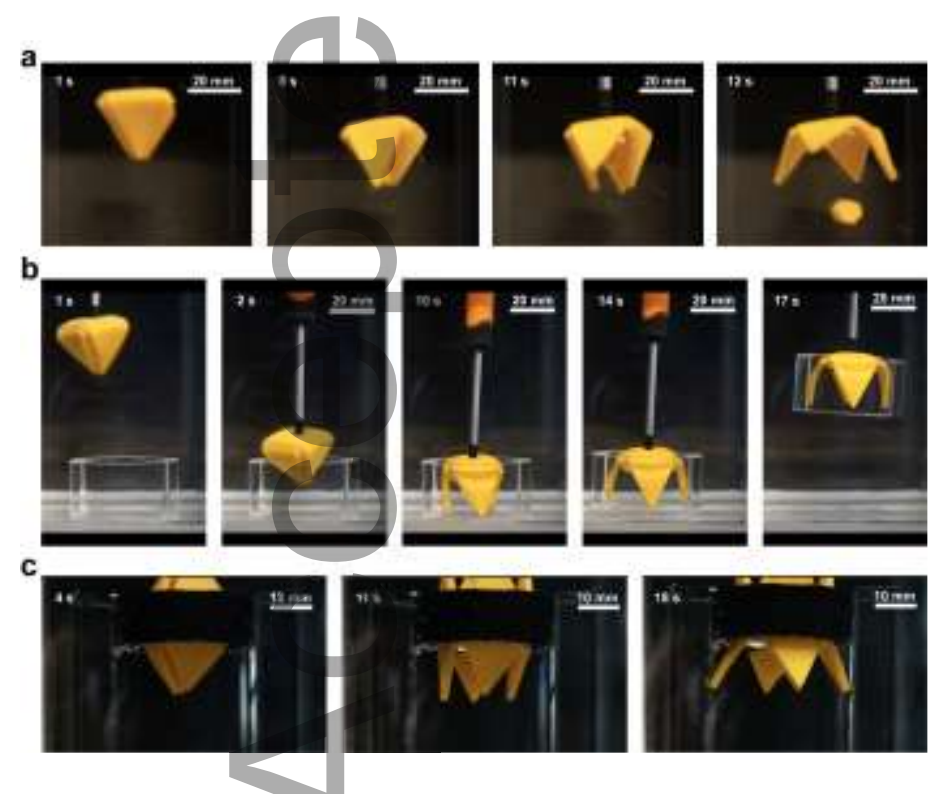

Figure 3. The application of the origami structures to (a) releases an encapsulated material, (b) grasping an object, and (c) blocking a gap.

\section{Conclusion}

We propose a very simple approach for the development of smart materials for various applications such as in biomedical engineering, aerospace, smart textiles, and advanced assembly techniques. Overall, the results below were obtained in brief:

- The activation temperature has a superior role in shape recovery since it can guarantee a full shape recovery and improves the rate of recovery. 
- Total thickness is the second important factor as it can effectively influence the reaction time delays, therefore realizing a sequential sequence of unbendings.

- As the layer height, regarding the layer by layer manufacturing of origami structures, is increased, the shape recovery ratio was increased too and the unfolding rate of the object during recovery was enhanced as well.

- Nozzle temperature is less influential in shape recovery compared to the other factors, however, it can significantly affect the mechanical properties of the 3D printed structures.

\section{Acknowledgment}

The authors thank Mr. Atabak Rahimzadeh for the support in the graphical analysis.

\section{References}

1. Huang, S.H., et al., Additive manufacturing and its societal impact: a literature review. The International Journal of Advanced Manufacturing Technology, 2012. 67(5-8): p. 1191-1203.

2. Stansbury, J.W. and M.J. Idacavage, 3D printing with polymers: Challenges among expanding options and opportunities. Dent Mater, 2016. 32(1): p. 54-64.

3. Mehrpouya, M., et al., The potential of additive manufacturing in the smart factory industrial 4.0: A review. Applied Sciences, 2019. 9(18): p. 3865.

4. Kuang, X., et al., Advances in 4D Printing: materials and applications. 2019. 29(2): p. 1805290.

5. Gisario, A., et al., Metal additive manufacturing in the commercial aviation industry: A review. Journal of Manufacturing Systems, 2019. 53: p. 124-149.

6. Janbaz, S., M. McGuinness, and A.A. Zadpoor, Multimaterial control of instability in soft mechanical metamaterials. Physical Review Applied, 2018. 9(6): p. 064013.

7. Janbaz, S., et al., Ultra-programmable buckling-driven soft cellular mechanisms. Materials Horizons, 2019. 6(6): p. 1138-1147.

8. Bhushan, B. and M.J.M.T. Caspers, An overview of additive manufacturing (3D printing) for microfabrication. 2017. 23(4): p. 1117-1124.

9. AHMAD, A., et al., Rapid prototyping for assembly training and validation. 2015. 48(3): $\mathrm{p}$. 412-417.

10. Janbaz, S., H. Weinans, and A.A. Zadpoor, Geometry-based control of instability patterns in cellular soft matter. RSC advances, 2016. 6(24): p. 20431-20436.

11. Nguyen, T.D., E. Jankowski, and S.C. Glotzer, Self-assembly and reconfigurability of shape-shifting particles. ACS nano, 2011. 5(11): p. 8892-8903.

12. Janbaz, S., R. Hedayati, and A. Zadpoor, Programming the shape-shifting of flat soft matter: from self-rolling/self-twisting materials to self-folding origami. Materials Horizons, 2016. 3(6): p. 536-547.

13. Skylar Tibbits. The Emergence 4D Printing. 2013; Available from: https://www.ted.com/talks/skylar_tibbits_the_emergence_of_4d_printing?language=en.

14. Yang, H., et al., 3D Printed Photoresponsive Devices Based on Shape Memory Composites. Advanced Materials, 2017. 29(33).

15. Gladman, A.S., et al., Biomimetic 4D printing. Nat Mater, 2016. 15(4): p. 413-8.

16. Ding, Z., et al., Direct 4D printing via active composite materials. 2017. 3(4): p. e1602890.

17. Nadgorny, M., et al., Three-Dimensional Printing of $\mathrm{pH}$-Responsive and Functional Polymers on an Affordable Desktop Printer. ACS Appl Mater Interfaces, 2016. 8(42): p. 28946-28954.

18. Bobbert, F., et al., Russian doll deployable meta-implants: Fusion of kirigami, origami, and multi-stability. Materials \& Design, 2020: p. 108624.

19. Ge, Q., et al., Multimaterial 4D printing with tailorable shape memory polymers. Scientific reports, 2016. 6: p. 31110.

This article is protected by copyright. All rights reserved 


\section{WILEY-VCH}

20. Zarek, M., et al., 3D printing of shape memory polymers for flexible electronic devices. Advanced Materials, 2016. 28(22): p. 4449-4454.

21. van Manen, T., S. Janbaz, and A.A. Zadpoor, Programming 2D/3D shape-shifting with hobbyist 3D printers. Materials horizons, 2017. 4(6): p. 1064-1069.

22. Zhou, Y., et al., From 3D to 4D printing: approaches and typical applications. Journal of Mechanical Science and Technology, 2015. 29(10): p. 4281-4288.

23. Yang, W., et al., Advanced Shape Memory Technology to Reshape Product Design, Manufacturing and Recycling. Polymers, 2014. 6(8): p. 2287-2308.

24. Inoue, K., M. Yamashiro, and M.J.J.o.A.P.S. Iji, Recyclable shapememory polymer: Poly (lactic acid) crosslinked by a thermoreversible Diels-Alder reaction. 2009. 112(2): p. 876885.

25. Leist, S.K., et al., Investigating the shape memory properties of 4D printed polylactic acid (PLA) and the concept of 4D printing onto nylon fabrics for the creation of smart textiles. Virtual and Physical Prototyping, 2017. 12(4): p. 290-300.

26. Johnson, M., et al., Fabricating biomedical origami: a state-of-the-art review. International journal of computer assisted radiology and surgery, 2017. 12(11): p. 2023-2032.

27. Whitesides, G.M., Soft robotics. Angewandte Chemie International Edition, 2018. 57(16): p. 4258-4273.

28. Lee, D.-Y., et al., Origami wheel transformer: A variable-diameter wheel drive robot using an origami structure. Soft robotics, 2017. 4(2): p. 163-180.

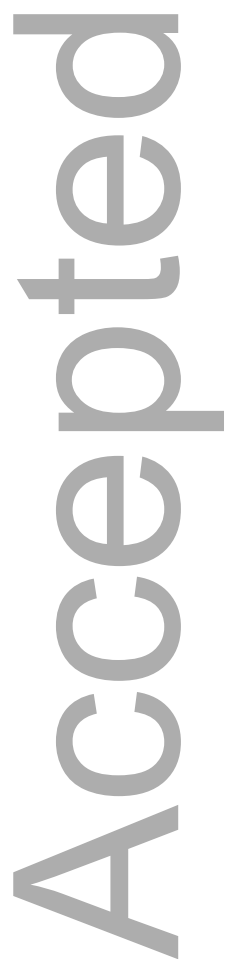

This article is protected by copyright. All rights reserved 


\section{WILEY-VCH}

\section{TOC Figure}

FDM printing of shape memory polymer like PLA can be applied in the fabrication of highly complex structures to plan shape recovery properties. The manual programming of origami structures is a simple solution to simply organize the desired deformations for various applications such as on-demand releases of material, smart grasping, and fixation.
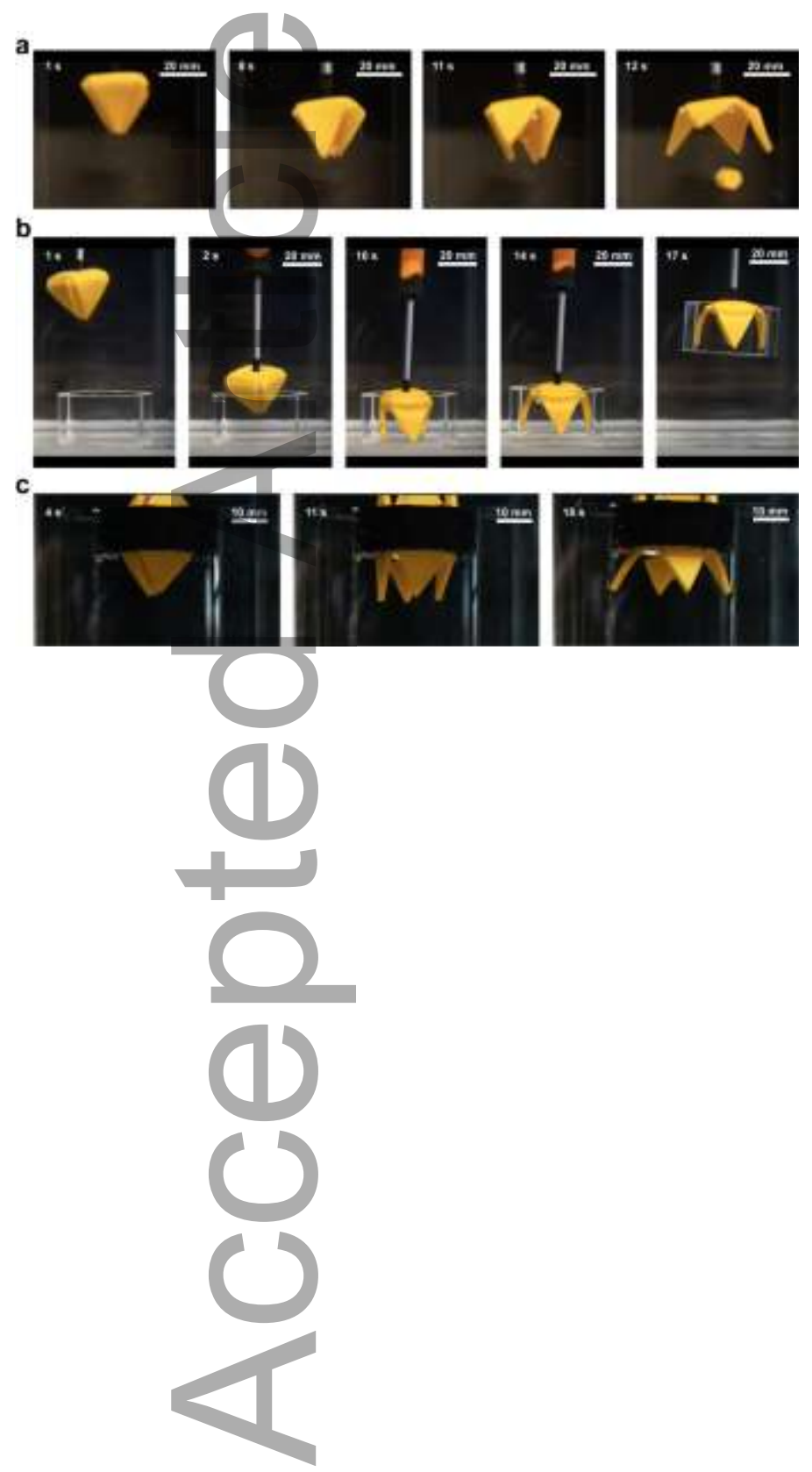

This article is protected by copyright. All rights reserved 